\title{
Evidence for GeV Cosmic Rays from White Dwarfs in the Local Cosmic Ray Spectra and in the Gamma- ray Emissivity of the Inner Galaxy
}

\author{
Tuneyoshi KAMAE' \\ U. of Tokyo/Physics and Stanford/SLAC \\ 7-3-1 Hongo, Bunkyo-ku, Tokyo, 113-0033, Japan \\ E-mail: kamaedslac.stanford.edu
}

Shiu-Hang LEE

Kyoto University/Astronomy

Kitashirakawa-Oiwake-cho, Sakyo-ku, Kyoto 606-8502, Japan

\section{Kazuo MAKISHIMA}

Makishima Cosmic Radiation Lab, RIKEN,

2-1 Hirosawa, Wako, Saitama 351-0198 Japan

\section{Shinpei SHIBATA}

Department of Physics, Graduate School of Science, Yamagata University

1-4-12, Kojirakawa-machi, Yamagata City, Yamagata 990-8560, Japan

\section{Toshikazu SHIGEYAMA}

Research Center for the Early Universe, University of Tokyo

7-3-1 Hongo, Bunkyo-ku, Tokyo, 113-0033, Japan

Recent hard X-ray observations found that electrons are accelerated in magnetic white dwarfs (WDs). Detection of GeV gamma rays from novae by Fermi-LAT infers that protons are accelerated to hundreds of $\mathrm{GeV}$ there. These facts motivated us to search for the cosmic rays (CRs) from historic outbursts of WDs accumulated in the local bubble around us. We propose CR model spectra at the heliopause including the local CRs from historic WD outbursts. The total CR spectra are assumed to consist of these and the Galactic components deduced from Fermi-LAT $\gamma$-ray observations. The two components are fitted to reproduce the Voyager- 1 spectra and the high-energy CR data on/near Earth when summed, species by species. We find that a common local spectral shape and simple power-law Galactic spectral reproduce all nuclear CR spectra at the heliopause. The hardening of the nuclear CRs is found to be caused by the roll-down of the soft local WD CRs at around $\sim 300 \mathrm{GeV}$. The WD CRs induce a hump in $\gamma$-ray emissivity in the GeV range. Such a hump is found in the inner Galaxy indicating that the fluxes of CRs from WD outbursts CRs $\sim 2.5$ times higher there than inside the local bubble.

7th Fermi Symposium 2017

15-20 October 2017

Garmisch-Partenkirchen, Germany

\footnotetext{
${ }^{1}$ Speaker 


\section{Introduction}

The CR spectral shapes have been analyzed extensively in the literature in the past several years. Most studies have focused on the positron-electron ratio measurements as summarized in ref [1]. In this short presentaation we focus on the electron, proton and nuclear CR spectra at the heliopause between the Voyager 1 energy and a few $\mathrm{TeV}$ and aim at separating the CRs from historic WD outbursts (electrons from magnetic WDs and protons from novae) accumulated in the local bubble. Our model differs from the prior works in the following points.

- Voelk and Berezhko [2] found a surplus in the low energy spectra. Tomassetti [3] fitted the proton and helium spectra as a sum of sub-TeV and multi-TeV components. Neither associated the low energy CRs with local WD outbursts accumulated in the local bubble.

- No prior work fitted the local proton and nuclear CR spectra with one common spectral shape as done in this work.

- Our model identifies local WDs to be a major source of sub-TeV CRs, quantifies their fluxes, and allows one to compare the CR fluxes from WDs in Galaxy regions.

We note that the WD accelerators detected in the hard X-ray and gamma-ray bands are not within the local bubble. They are assumed, however, to represent magnetized WDs in the bubble and historic novae occurred in the bubble over the CR trapping lifetime of $10^{6}$ to $10^{7}$ years $[4,5]$.

\section{Model spectra for Galactic proton and electron at the heliopause}

Our knowledge on CR spectra comes from near-Earth observations except for those measured by Voyager-1 at the heliopause. CR spectra are known to be distorted along the propagation path from the Galactic space to the heliopause and from the heliopause to Earth. To simplify the spectral model we reducing the parameters to minimum as described below:

- We start with the Galactic spectra for electrons and protons deduced from the diffuse $\gamma$-ray emissivity in the local Galaxy (latitude $10<|b|<70 \mathrm{deg}$ ) given by Casandjian [6]. The shapes (not normalized) are shown in fig 1 by the black solid and dashed lines.

- The proton spectra described above were not constrained by the Voyager 1 data $[7,8]$. We define our Galactic proton spectra on the shapes of Casandjian [6] and CR data on Earth at $\mathrm{E}>100 \mathrm{GeV}$ and extend downward to the Voyager 1 data points in in polynomial regression of degree 6 or less with the variable set to the logarithm of kinetic energy, $\log _{10}(\mathrm{E}[\mathrm{GeV}])$ for $\mathrm{E}<400 \mathrm{GeV}$. For $\mathrm{E}>400 \mathrm{GeV}$ the electron spectrum is forced to follow the HESS data [9] and the proton spectrum is constrained to power-law with index -2.7 .

\section{Model spectra for local electron and proton CRs from WD outbursts}

Terada et al. [10] found synchrotrom hard X-ray and concluded that electrons are accelerated in a magnetic white dwarfs (WDs), AE Aquarii, to energies higher than $\sim 10 \mathrm{GeV}$. Geng, Zhang, \& Huang [11] analyzed radiation from another magnetic WD, AR Scorpii, detected by Marsh et al. [12] and predicted that the underlying electron spectrum has a broken PL shape. Oruru and Meintjes [13] studied the broad-band spectrum of AE Aquarii taken with Suzaku, Chandra, and Swift to find that the accelerated electrons reach $\sim 100 \mathrm{GeV}$. Based on these results, we build a model spectrum for the electron CR in the local bubble starting with a broken PL shape.

The local proton spectrum from WD outburstss was extracted from the $\gamma$-ray spectrum observed at V407 Cygnii 2010 [14]. The spectral shape was predicted to be of single PL shape with index $=2.15^{+0.45}-0.28$ and cut off exponentially at $32^{+85}{ }_{-8} \mathrm{GeV}$. We chose the PL index to -2.2 and to be cut-off at $150 \mathrm{GeV}$ in the initial trial. This form is reshaped in a polynomial regression until the heliopause data points are reproduced when summed with the Galactic proton spectrum. The results are shown in fig 1 . The black lines are the spectra deduced from $\gamma$-ray emissivity by Casandjian [6]. The initial local CR spectra are reshaped so that the sum with the respective Galactic components will reproduce the spectra at the heliopause as described below.

\section{Total CR spectra at the heliopause}

The fitted sums of the Galactic and local CR spectral models are shown in fig 2 by lines marked 
with "tot." We note the absolute flux of the Galactic component is determined by fitting the model spectra by polynomial curves of degree 6 or less to the Voyager-1 points and the high energy points $(E>100 \mathrm{GeV})$. The Galactic proton spectrum is set to a power-law shape with index -2.7 for $\mathrm{E}>400 \mathrm{GeV}$. Our total proton $\mathrm{CR}$ spectrum shown in figure 2 resembles to that of Casandjian [6] but differs for $\mathrm{E}<10 \mathrm{GeV}$ because we included the Voyager 1 data.

We note that the data points from Voyager-1 demand the local WD component to dominate over the Galactic component below a few GeV. This is in agreement with the claims of the "excess" by Lee et al. [15]. For $\mathrm{E}>1 \mathrm{TeV}$ ), the spectral cut-off in the HESS data [9] dictates.

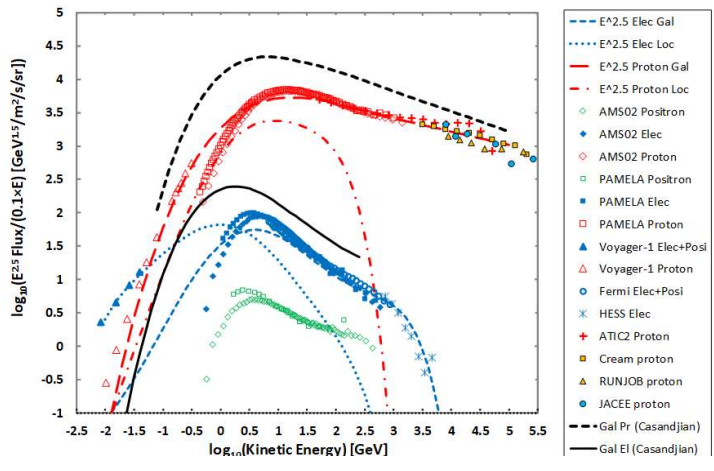

Fig 1: Gal electron/proton spectra deduced from $\gamma$-ray emissivity [6] (blk lines) and our fitted spectra for Gal/local elec/proton CRs (blue for electron and red for proton).

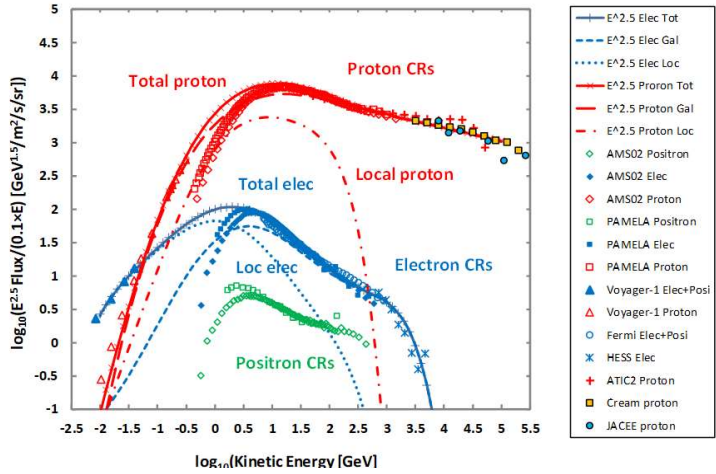

Fig 2: Total, Gal, local CR spectra: elec (blue lines) and proton (red lines). Solid with marks for total, dashed lines for Galactic, dotted for elec local and dot-dsh for pr local.

\section{Extension of our modeling to nuclear CR spectra at the heliopause}

Since there is no information about nuclear CR spectrum produced in WD outbursts, we start with a simple assumption that all local nuclear CRs have the same spectral shape as the proton local CR. The polynomial fitting is repeated and the final model spectra are obtained as shown in fig 3. They reproduce the data by Voyager-1 [8] and AMS02 [16] quite well. The PL index of the nuclear Galactic CR spectra are tweaked to fit the data for E/nucleon $>400 \mathrm{GeV}$.

\section{GeV-hump in the diffuse Fermi-LAT gamma-ray spectra}

Recent studies strongly suggest that WDs are contributing to the Galactic ridge hard X-ray emission [17]. Motivated by these findings, we search the sign of the $\gamma$-rays produced the local CRs from WD outbursts. The $\gamma$-rays from $\pi^{0}$ generated in the CR-ISM interaction are known to populate an energy range about one-tenth of the energy range per nucleon of proton and nuclear CRs. So a hump-like spectrum is expected in the $\mathrm{GeV}$ range. After comparing the diffuse $\gamma$-ray emissivity in the Galaxy [18], we found a hump in the inner Galaxy $(\mathrm{R}<1.5 \mathrm{kpc})$.

\section{Conclusions}

- The "hardening" in the nuclear CR spectra is due to roll-down of the local CRs from WD outbursts accumulated in the local bubble at around $300 \mathrm{GeV} / \mathrm{n}$. Domination of the harder Galactic component (PL index 2.51) above the energy mimics the spectral hardening.

- Interaction of the CRs from WD outbursts with ISM produces a hump in $\gamma$-ray emissivity in the $\mathrm{GeV}$ range. Such a hump is seen in the Fermi-LAT data from the inner Galaxy [18].

\section{References}

[1] Accardo, L., Aguilar, M., Aisa, D., Alpat, B., Alvino, A., Ambrosi, G., et al. "High Statistics Measurement of the Positron Fraction in Primary Cosmic Rays of 0.5-500 GeV with the Alpha Magnetic Spectrometer on the International Space Station”, Phys. Rev. Lett. 113 (2014)121101

[2] Voelk, H. J., \& Berezhko, E. G. "On the Fermi Large Area Telescope Surplus of Diffuse Galactic Gamma-Ray Emission”, Astrophys. J777 (2013) 149

[3] Tomassetti, N. "Testing universality of cosmic-ray acceleration with proton/helium data from AMS and Voyager-1", AdSpR 60 (2017) 815 


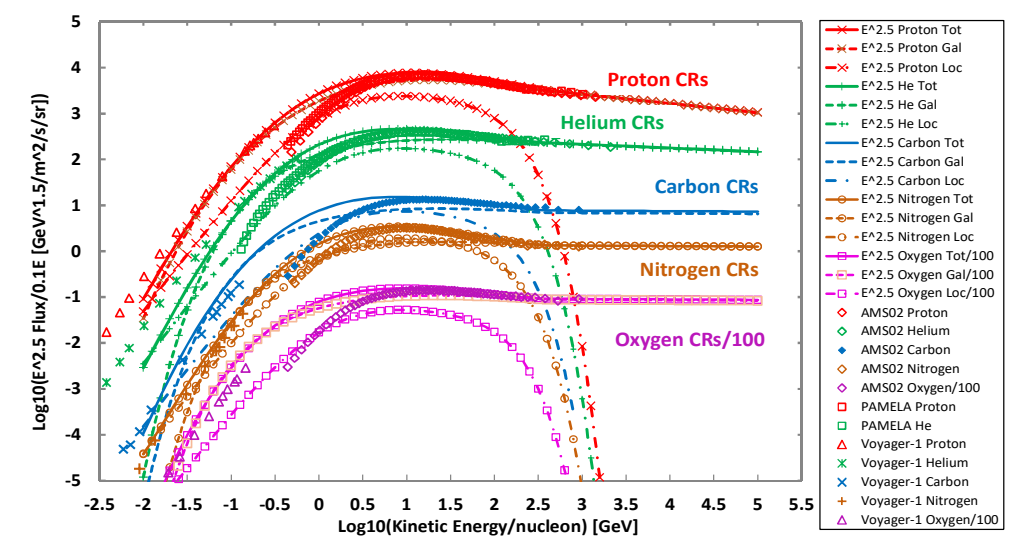

Fig 3: Total (solid lines with or w/o marks), Galactic (dashed lines with or w/o marks), and local WD outbursts contribution (dot-dash with or w/o marks) for nuclear CR spectra. Proton: red; He: green; $\mathrm{C}$ : blue; N: brown; $\mathrm{O}$ (x 1/100): purple.

[4]Strong, A. W., Moskalenko, I. V., \& Ptuskin, V. S. “Cosmic-Ray Propagation and Interactions in the Galaxy” Annu. Rev. Nucl. Part. Sci., 57 (2007) 285

[5] Erlykin, A. D., Machavariani, S. K., \& Wolfendale, A. W. "The Local Bubble in the interstellar medium and the origin of the low energy cosmic rays", AdSpR, 59 (2017)748

[6] Casandjian, J.-M. "Local H I Emissivity Measured with Fermi-LAT and Implications for CosmicRay Spectra”, Astrophys. J. 806 (2015) 240

[7] Stone, E. C., Cummings, A. C., McDonald, F. B., Heikkila, B. C., Lal, N., \& Webber, W. R. "Voyager 1 observes low-energy Galactic cosmic rays in a region depleted of heliospheric ions", Science 341 (2013) 150

[8] Cummings, A. C., Stone, E. C., Heikkila, B. C., Lal, N., Webber, W. R.; Jóhannesson, G. et al. "Galactic Cosmic Rays in the Local Interstellar Medium: Voyager 1 Observations and Model Results" Astrophys. J. 831 (2016) 18

[9] Aharonian, F., Akhperjanian, A. G., Barres de Almeida, U., Bazer-Bachi, A. R., Becherini, Y., Behera, B. et al., "Energy Spectrum of Cosmic-Ray Electrons at TeV Energies" Phs. Rev. Lett. 101 (2008) 1104

[10] Terada, Y., Hayashi, T., Ishida, M., Mukai, K., Dotani, T., Okada, S. et al., "Suzaku Discovery of Hard X-Ray Pulsations from a Rotating Magnetized White Dwarf, AEAquarii", PASJ, 60 (2008) 387

[11] Geng, J.-J., Zhang, B., Huang, \& Y.-F. “A model of white dwarf pulsar AR Scorpii”, Astrophys. J. 831 (2016) L10

[12] Marsh, T. R., G“ansicke, B. T., H“ummerich, S., Hambsch, F.-J., Bernhard, K., Lloyd, C. et al. “A radio pulsing white dwarf binary star", Nature 537 (2016) 374

[13] Oruru, B., \& Meintjes, P. J. "X-ray characteristics and the spectral energy distribution of AE Aquarii" MNRAS 421 (2012) 1557

[14] Abdo, A. A., Ackermann, M., Ajello, M., Atwood, W. B., Baldini, L., Ballet, J. et al. "Gamma-Ray Emission Concurrent with the Nova in the Symbiotic Binary V407 Cygni”, Science 329 (2010) 817

[15] Lee, S.-H., Kamae, T., Baldini, L., Giordano, F., Grondin, M.-H., Latronico, L. et al. "Explaining the cosmic-ray $e^{+} /(e+e+)$ and pbar/p ratios using a steady-state injection model”, Astropart. Phys. 35 (2011) 211

[16] Yan, Q. et al. (AMS02) "Precision Measurement of the Proton and Nuclei Fluxes with AMS Experiment” XSCRC (March 2017, CERN)

[17] Yuasa, T., Makishima, K., \& Nakazawa, K. "Broadband Spectral Analysis of the Galactic Ridge XRay Emission”, Astrophys. J. 753 (2012) 129

[18] Acero, F., Ackermann, M., Ajello, M., Albert, A., Baldini, L., Ballet, J. et al. "Development of the Model of Galactic Interstellar Emission for Standard Point-source Analysis of Fermi Large Area Telescope Data”, Astrophys J. Suppl, 223 (2016) 26 